\title{
A THEOREM ON ANALYTIC FUNCTIONS OF A REAL VARIABLE
}

BY R. P. BOAS, JR.

1. Introduction. Let $f(x)$ be a function of class $C^{\infty}$ on $a \leqq x \leqq b$. At each point $x$ of $[a, b]$ we form the formal Taylor series of $f(x)$,

$$
\sum_{k=0}^{\infty} \frac{f^{(k)}(x)}{k !}(t-x)^{k} .
$$

This series has a definite radius of convergence, $\rho(x)$, zero, finite, or infinite, given by $1 / \rho(x)=\varlimsup_{\lim _{k \rightarrow \infty}}\left|f^{(k)}(x) / k !\right| 1 / k$. The function $f(x)$ is said to be analytic at the point $x$ if the Taylor development of $f(x)$ about $x$ converges to $f(t)$ over a neighborhood $|x-t|<c, c>0$, of the point; $f(x)$ is analytic in an interval if it is analytic at every point of the interval.

Pringsheim stated the following theorem.*

TheOREm A. If there exists a number $\delta>0$ such that $\rho(x) \geqq \delta$ for $a \leqq x \leqq b, f(x)$ is analytic in $[a, b]$.

However, Pringsheim's proof of the theorem is not rigorous. The purpose of this note is to establish this theorem, and, in connection with the proof, a companion theorem of considerable interest in itself.

TheOREM B. If $\rho(x)>0$ for $a \leqq x \leqq b$ (that is, if the Taylor development of $f(x)$ about each point converges in some neighborhood of the point), the points at which $f(x)$ is not analytic form at most a nowhere dense closed set.

Theorem B is, in a certain sense, the best possible, since by a theorem of $\mathrm{H}$. Whitney $\dagger$ there exist functions satisfying the

* A. Pringsheim, Zur Theorie der Taylor'schen Reihe und der analytischen Funktionen mit beschränkten Existenzbereich, Mathematische Annalen, vol. 42 (1893), p. 180.

$\dagger \mathrm{H}$. Whitney, Analytic extensions of differentiable functions defined in closed sets, Transactions of this Society, vol. 36 (1934), pp. 63-89. I am indebted to Dr. Whitney for calling my attention to this paper. 
conditions of Theorem B and having the points of an arbitrary nowhere dense closed set as singular points.

Theorem B can also be stated in the following equivalent form.

Theorem $\mathrm{B}^{\prime}$. If $f(x)$ is of class $C^{\infty}$ on $[a, b]$ and analytic at no point of $[a, b]$, there must exist an everywhere dense set of points, $G$, on $[a, b]$ such that the Taylor development of $f(x)$ about each point of $G$ is divergent.

We shall need the following lemma.*

Lemma. If $H$ is a perfect point set on the interval $[\alpha, \beta]$, and if $H=\sum_{n=0}^{\infty} H_{n}$, where the $H_{n}$ are enumerable in number, there exist a value $n_{0}$ of $n$ and a sub-interval $\left[\alpha_{0}, \beta_{0}\right]$ such that $H_{n_{0}}$ is dense in $H \cdot\left[\alpha_{0}, \beta_{0}\right]$.

2. Proof of Theorem B. For each $x$ in $[a, b]$,

$$
\frac{1}{\rho(x)}=\varlimsup_{n \rightarrow \infty}\left|\frac{f^{(n)}(x)}{n !}\right|^{1 / n}<\infty .
$$

This implies that there exists a finite function $\mu(x)$ such that

$$
\left|\frac{1}{n !} f^{(n)}(x)\right|^{1 / n} \leqq \mu(x), \quad(n=1,2, \cdots),
$$

or,

$$
\left|f^{(n)}(x)\right| \leqq n ![\mu(x)]^{n}, \quad(n=1,2, \cdots) .
$$

Let $E_{k}$ be the set (not necessarily non-empty) of points $x$ such that

$$
k \leqq \mu(x)<k+1, \quad(k=0,1,2, \cdots) .
$$

It is clear that $[a, b]=\sum_{=0}^{\infty} E_{k}$. By the lemma, there is a subinterval $[\alpha, \beta]$ and an integer $k_{0}$ such that $E_{k_{0}}$ is dense in $[\alpha, \beta]$. For every point of $E_{k_{0}} \cdot[\alpha, \beta]$,

$$
\left|f^{(n)}(x)\right| \leqq n ![\mu(x)]^{n}<n !\left(k_{0}+1\right)^{n}, \quad(n=1,2, \cdots) .
$$

For every point of $[\alpha, \beta] \cdot C\left(E_{k_{0}}\right),(1)$ holds by continuity. That

* See Lebesgue, Leçons sur l'Intégration, 2d ed., 1928, p. 203. See also S. Banach, Théorie des Opérations Linéaires, 1932, p. 14 (Theorem 2). 
is, (1) holds uniformly in $[\alpha, \beta] .^{*}$ But this is a well known sufficient condition for $f(x)$ to be analytic in $[\alpha, \beta]$. The same reasoning applies to any sub-interval of $[a, b]-[\alpha, \beta]$; thus in any sub-interval there is a further sub-interval in which $f(x)$ is analytic. The points at which $f(x)$ is not analytic thus form a nowhere dense set, which is obviously closed.

3. Proof of Theorem A. Assume the theorem false; we shall obtain a contradiction. We have, then, a non-empty set $H$ of points where $f(x)$ is not analytic, and by Theorem $\mathrm{B}, H$ is closed and nowhere dense.

We first show that $H$ is perfect. Suppose that $H$ contained an isolated point $X$. The function $f(x)$ is continuous with all derivatives at $X ; f(x)$ is analytic in each of the intervals $X-h<x<X$ and $X<x<X+h$, for some $h>0$, and can be extended analytically across the point $X$ in both directions. It follows immediately that $f(x)$ is analytic at $X$, so that $X$ is not a singular point. $H$ being perfect, from now on we shall confine our attention to an interval $\left[a_{1}, b_{1}\right]$ such that $b_{1}-a_{1}<\delta / 4$ and $\left[a_{1}, b_{1}\right]$ contains a perfect subset $E$ of $H$.

Since by hypothesis

$$
\varlimsup_{n \rightarrow \infty}\left|\frac{f^{(n)}(x)}{n !}\right|^{1 / n}=\frac{1}{\rho(x)} \leqq \frac{1}{\delta}
$$

for every point of $\left[a_{1}, b_{1}\right]$, it follows that for each $x$ in $E$ there is an integer $N_{x}$ such that

hence

$$
\left|\frac{1}{n !} f^{(n)}(x)\right|^{1 / n}<\frac{2}{\delta}, \quad\left(n \geqq N_{x}\right) ;
$$

$$
\left|f^{(n)}(x)\right| \leqq n ! \lambda^{n}, \quad\left(\lambda=2 / \delta, n \geqq N_{x}\right) .
$$

Let $E_{k}$ be the set of points of $E$ for which $N_{x}=k$. By the lemma, there exist a sub-interval $[\alpha, \beta]$ and a value $k_{0}$ of $k$ such that $E_{k_{0}}$ is dense in $E \cdot[\alpha, \beta]$. For $x$ in $E_{k_{0}} \cdot[\alpha, \beta],(2)$ holds for $n \geqq k_{0}$. For $x$ in $\left(E-E_{k_{0}}\right) \cdot[\alpha, \beta]$, (2) holds for $n \geqq k_{0}$, by continuity. Thus (2) holds uniformly for $x$ in $E \cdot[\alpha, \beta], n \geqq k_{0}$.

* This fact can be obtained as a special case of a general theorem in the theory of operations, which is established by similar reasoning; see S. Banach, op. cit., p. 19 (Theorem 11). 
Let $\left(x_{0}, y_{0}\right)$ be a complementary interval of the nowhere dense set $E \cdot[\alpha, \beta]$. Then the Taylor series

$$
\sum_{k=0}^{\infty} \frac{f^{(k)}\left(x_{0}\right)}{k !}\left(x-x_{0}\right)^{k}
$$

converges to $f(x)$ for $x_{0} \leqq x<y_{0}$. This follows at once from the facts that $f^{(k)}(x)$ is continuous on $x_{0} \leqq x<y_{0}$ for $k=0,1,2, \cdots$, and that $y_{0}-x_{0}<\delta / 4<\delta$.

Define an auxiliary function $\phi(x)=\left(\beta_{1}-\alpha\right) /\left(\beta_{1}-x\right)$, where $\delta / 4>\beta_{1}-\beta>\beta-\alpha>0$. The function $\phi(x)$ is analytic on $[\alpha, \beta]$ and is represented over the whole of $[\alpha, \beta]$ by its Taylor development about any point of $[\alpha, \beta]$. We have

$$
\begin{aligned}
\phi^{(k)}(x)=\frac{\left(\beta_{1}-\alpha\right) \cdot k !}{\left(\beta_{1}-x\right)^{k+1}} \geqq & \frac{\left(\beta_{1}-\alpha\right) \cdot k !}{\left(\beta_{1}-\alpha\right)^{k+1}} \geqq k ! \lambda{ }^{k}, \\
& (k=0,1,2, \cdots ; \alpha \leqq x \leqq \beta) .
\end{aligned}
$$

Now form $\psi(x)=\phi(x)-f(x)$. The function $\psi(x)$ is represented by its Taylor development about $x_{0}$ for $x_{0} \leqq x<y_{0}$; for $n \geqq k_{0}$, $\psi^{(n)}\left(x_{0}\right) \geqq 0$ and $\psi^{(n)}\left(y_{0}\right) \geqq 0$ by $(2)$. Hence for $n \geqq k_{0}, \psi^{(n)}(x) \geqq 0$ for $x_{0} \leqq x \leqq y_{0}$, since we may differentiate a power series termwise any number of times in the interior of its interval of convergence, so that $\psi^{(n)}(x)$ is represented over $x_{0} \leqq x<y_{0}$ by a series of non-negative terms, for $n \geqq k_{0}$. This reasoning applies to any complementary interval of $E \cdot[\alpha, \beta]$, with the same function $\psi(x)$. Hence $\psi^{(n)}(x) \geqq 0$ for $\alpha \leqq x \leqq \beta, n \geqq k_{0}$. By a well known theorem of S. Bernstein, $\psi^{\left(k_{0}\right)}(x)$ is analytic for $\alpha \leqq x \leqq \beta$, and consequently $\psi(x)$ is analytic in the same interval. But then $f(x)$ $=\phi(x)-\psi(x)$ is analytic in $[\alpha, \beta]$, contrary to the hypothesis that $H$ was not an empty set. Hence $H$ is an empty set, and the theorem is proved.

HARVARD UNIVERSITY 